\section{JTI}

JOURNAL OF

TRAUMA AND INJURY

Received: September 15, 2020

Revised: November 10, 2020

Accepted: November 18, 2020

\section{Correspondence to}

Sung Won Jung, M.D., Ph.D.

Department of Surgery, Ilsan Paik Hospital, Inje University College of Medicine,

170 Juhwa-ro, Ilsanseo-gu, Goyang 10380, Korea

Tel: +82-31-910-7114

Fax: +82-31-910-7114

E-mail: sungwon94@naver.com

\title{
The Prognosis of Traumatic Small Bowel Injury Accompanied by Liver Injury
}

Yu Seong Noh, M.D., Sung Won Jung, M.D., Ph.D., Tae Gil Heo, M.D., Ph.D., Pyong Wha Choi, M.D., Ph.D., Jae Il Kim, M.D., M.S., Heung Man Jun, M.D., Ph.D., Yong Chan Shin, M.D., M.S., Sung Min Jung, M.D., M.S., Eun Hae Um, M.D., M.S.

Department of Surgery, Ilsan Paik Hospital, Inje University College of Medicine, Goyang, Korea

Purpose: The aim of this study was to elucidate the prognosis, and other clinical features, such as time to surgery and the amount of transfusion, of small bowel injury (SBI) accompanied by liver injury (LI).

Methods: We investigated 221 patients with SBI who visited an emergency center from October 2000 to March 2019. We excluded patients with injuries that directly led to mortality, and the remaining 149 patients were divided into the SBI alone (SBI-A) group and the SBI accompanied by LI (SBI-LI) group. Data were collected for preoperative and surgical outcome variables, and the treatment results were compared between groups. Results: The SBI-LI group had a higher mortality rate than the SBI-A group (22.4\% vs. $14.3 \%$ ), but this difference was not statistically significant $(p=0.061)$. There were no significant differences between the SBI-A and SBI-LI groups, except for the amount of red blood cell (RBC) transfusion (SBI-A: $3.53 \pm 0.1$ vs. SBI-LI: $8.38 \pm 0.7$ packs, $p=0.035$ ) and the length of intensive care unit (ICU) stay (SBI-A: $6.7 \pm 0.2$ vs. SBI-LI: $11.1 \pm 0.5$ days, $p=0.047$ ).

Conclusions: The SBI-LI group required more RBC transfusions and longer ICU stays than the SBI-A group. SBI accompanied by LI may show higher mortality than SBI alone; however, since the difference was not statistically significant in the present study, larger-scale follow-up research is needed.

Keywords: Abdominal injuries; Intestines; Liver; Lacerations; Prognosis 


\section{INTRODUCTION}

The incidence of abdominal organ injuries is increasing due to traffic and industrial accidents [1]. In particular, small bowel injury (SBI) is frequently associated with blunt abdominal injury, occurring in $3 \%$ to $18 \%$ of cases [2], and it can be associated with peritonitis or sepsis, which leads to increased morbidity and mortality [3]. The diagnosis of SBI is difficult because its clinical signs and symptoms vary widely among patients. The possibility of delayed perforation also makes the diagnosis of SBI difficult. In multiple trauma patients, the diagnosis of SBI is challenging because the symptoms and signs of SBI are masked, a problem that is even further exacerbated in patients rendered unconscious by shock or traumatic brain injury [4].

The liver is also one of the most frequently injured organs in blunt abdominal trauma. Previous studies have reported a $16 \%$ incidence of liver injury (LI) in polytrauma patients [5]. Most blunt LIs can be treated by conservative management [6]. However, the management of high-grade LIs with hemodynamic instability is exceptionally difficult.

When SBI is accompanied by LI, the diagnosis and treatment can be more complex and challenging than when it is not. For example, in a patient with LI but not SBI, transcatheter angioembolization can be performed, whereas when LI accompanies SBI, diagnostic laparotomy needs to be considered.

Notably, SBI with LI can appear with different clinical features specific to SBI and LI, thereby affecting the diagnosis, management, and prognosis. However, insufficient evidence has been reported regarding SBI accompanied by LI. This study aimed to elucidate the prognosis, and other clinical features of SBI accompanied by LI, including time to surgery and the amount of red blood cell (RBC) transfusion.

\section{METHODS}

A retrospective cohort study including 221 blunt trauma patients was performed. These patients were diagnosed with SBI by emergency laparotomy at Ilsan Paik Hospital from October 2000 to March 2019. Patients whose cause of death was not SBI or LI $(n=72)$ were excluded from this study, which included eight cases of stabbing, 11 cases of cerebral hemorrhage, seven cases of pneumothorax, five cases of hemothorax, nine cases of pelvic fracture, six cases of major vessel injury, six cases of gastric injury, five cases of duodenal injury, and 15 cases of colon injury. Finally, a total of 149 patients were enrolled in this study. These 149 patients were divided into two groups: $91 \mathrm{pa}$ tients with SBI alone (SBI-A) and 58 patients with SBI

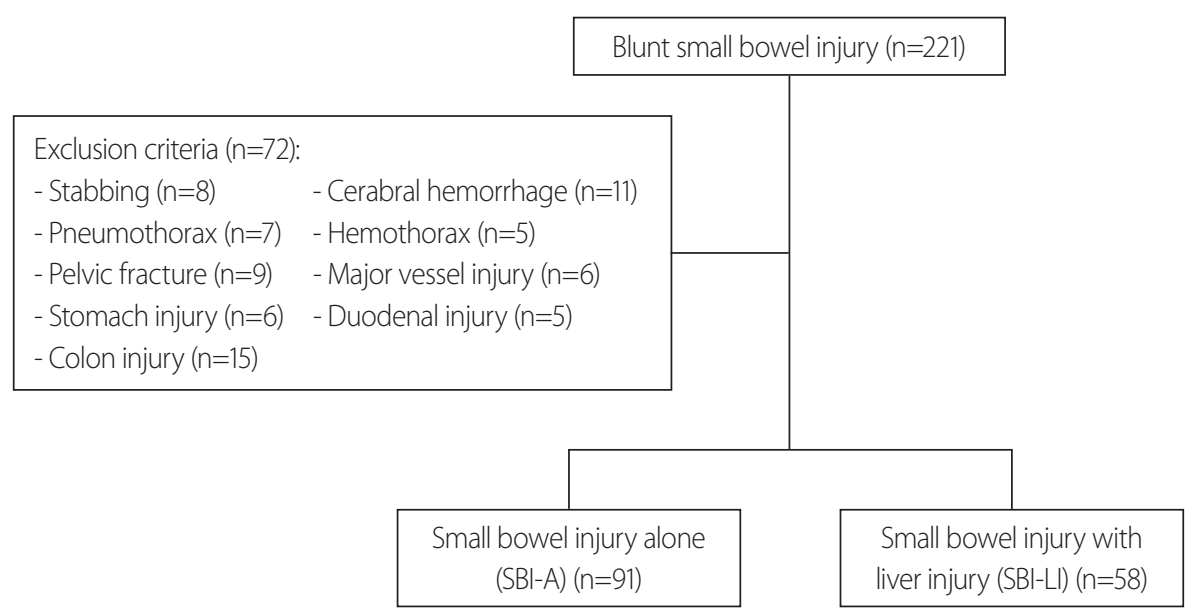

Fig. 1. Exclusion criteria: patients whose cause of death was not SBI or LI (eight cases of stabbing, 11 cases of cerebral hemorrhage, seven cases of pneumothorax, five cases of hemothorax, nine cases of pelvic fracture, six cases of major vessel injury, six cases of stomach injury, five cases of duodenal injury, and 15 cases of colon injury). SBI-A: small bowel injury alone, SBI-LI: small bowel injury accompanied by liver injury. 
accompanied by LI (SBI-LI) (Fig. 1). SBI and LI were diagnosed and classified by preoperative computed tomography and operative findings. LI was classified according to the American Association for the Surgery of Trauma (AAST) liver trauma classification system.

Information on demographic, preoperative, and surgical outcome variables was collected from patients' medical records. The demographic and preoperative variables included age, sex, underlying disease, trauma mechanisms, Abbreviated Injury Scale (AIS), presence of initial hypotension (initial systolic blood pressure $<90 \mathrm{mmHg}$ ), coexistence of LI, and grade of LI. The injury mechanisms were classified as follows: passenger traffic accidents (TAs), pedestrian TAs, falls, and others.

The surgical outcome variables included time to surgery, operation time, the amount of RBCs transfused during the operation, small bowel perforation, and type of surgery. The time to surgery was defined as the time taken from presenting at the emergency room to beginning the operation. The types of surgery included primary repair, small bowel resection and anastomosis, and bleeding control. Length of intensive care unit (ICU) stay, length of hospital stay, morbidity, and mortality were investigated

\section{Table 1. Demographics}

\begin{tabular}{|lccc|}
\hline & SBI-A (n=91) & SBI-LI (n=58) & $\boldsymbol{p}$-value \\
\hline Age & $49.2 \pm 8.2$ & $47.5 \pm 9.1$ & 0.149 \\
Male & $62(68.1)$ & $40(69.0)$ & 0.213 \\
Underlying disease & & & \\
Heart failure & $2(2.2)$ & $1(1.7)$ & 0.241 \\
Chronic renal failure & $4(4.4)$ & $2(3.4)$ & 0.521 \\
Liver cirrhosis & $7(7.7)$ & $4(6.9)$ & 0.385 \\
Chronic lung disease & $2(2.2)$ & $0(0.0)$ & 0.249 \\
Trauma mechanism & & & \\
Passenger TA & $30(33.0)$ & $16(27.6)$ & 0.218 \\
Pedestrian TA & $27(29.7)$ & $22(37.9)$ & 0.345 \\
Fall & $25(27.5)$ & $14(24.1)$ & 0.418 \\
Others & $9(9.9)$ & $6(10.3)$ & 0.311 \\
AlS & $14.3 \pm 5.7$ & $22.4 \pm 4.1$ & 0.041 \\
Initial hypotension & $23(25.3)$ & $19(32.8)$ & 0.069 \\
\hline
\end{tabular}

Values are presented as mean \pm standard deviation or number (\%). SBI-A: small bowel injury alone, SBI-Ll: small bowel injury accompanied by liver injury, TA: traffic accident, AIS: Abbreviated Injury Scale. as outcome variables in this study. Morbidity was categorized according to the Clavien-Dindo classification, and the mortality rate was defined based on in-hospital mortality.

The primary endpoint of this study was the mortality rate of each group. The secondary endpoints were the length of ICU stay, the length of hospital stay, and morbidity.

All continuous data were presented as mean \pm standard deviation, unless otherwise noted. The chi-square test or Fisher exact test was used for categorical or nominal variables. For continuous variables, the Student $t$-test was used. A $p$-value of $<0.05$ (two-tailed) was considered to indicate statistical significance. All statistical analyses were performed with SPSS version 19.0 for Windows (IBM Corp., Armonk, NY, USA).

\section{RESULTS}

The demographic and preoperative characteristics of each group are shown in Table 1. There were no statistically significant differences between the two groups, except in AIS scores, which were significantly higher in the SBILI group than in the SBI-A group (22.4 \pm 4.1 vs. $14.3 \pm 5.7$, $p=0.041)$. The number of patients with AAST LI grades I, II, III, IV, and V were 19 (32.7\%), 22 (37.9\%), 15 (25.8\%), $2(3.4 \%), 0(0 \%)$, respectively.

Regarding the surgery-related variables, there was no

Table 2. Surgery related variables

\begin{tabular}{|lccc|}
\hline & SBI-A (n=91) & SBI-LI (n=58) & $\boldsymbol{p}$-value \\
\hline Time to surgery (min) & $175 \pm 21.4$ & $140 \pm 18.1$ & 0.271 \\
Operation time (min) & $371 \pm 32.8$ & $415 \pm 30.7$ & 0.061 \\
RBC transfusion (pack) & $3.53 \pm 0.1$ & $8.38 \pm 0.7$ & 0.035 \\
Small bowel perforation & $49(53.8)$ & $36(62.1)$ & 0.382 \\
Operation & & & \\
Primary repair & $26(28.6)$ & $18(31.0)$ & 0.419 \\
Small bowel R \& A & $71(78.0)$ & $40(69.0)$ & 0.390 \\
Bleeding control & $11(12.1)$ & $6(10.3)$ & 0.201 \\
\hline
\end{tabular}

Values are presented as mean \pm standard deviation or number (\%). SBI-A: small bowel injury alone, SBI-LI: small bowel injury accompanied by liver injury, RBC: red blood cell, R \& A: resection and anastomosis. 
significant difference between the groups, except in the amount of RBC transfusion. The SBI-LI group had significantly more RBC packs transfused than the SBI-A group ( $8.38 \pm 0.7$ vs. $3.53 \pm 0.1$ packs, $p=0.035$; Table 2 ).

The only significant difference in the outcome variables between the two groups was found for the length of ICU stay, which was significantly longer in the SBILI group than in the SBI-A group ( $6.7 \pm 0.2$ vs. $11.1 \pm 0.5$ days, $p=0.047$; Table 3). The mortality rate of the SBI-LI group was higher than that of the SBI-A group (22.4\% vs. $14.3 \%)$, but this difference was not statistically significant $(p=0.061$; Table 3$)$.

\section{DISCUSSION}

The small bowel, which occupies the widest space of the hollow viscus, is the most frequently injured organ in hollow viscus injuries [4]. Several scenarios may lead to an SBI with abdominal blunt trauma. First, a shearing force can easily be applied due to acceleration and deceleration in fixed areas, such as the ligament of Treitz, the ileocecal valve, or mesenteric vessels. Second, compression between the abdominal wall and the vertebral column may crush the small bowel. Third, a sudden increase in the intraluminal pressure in the small bowel during abdominal blunt trauma may cause a bursting injury [2,7-9].

With the increasing frequency of TAs, abdominal blunt trauma caused by motor vehicle accidents has come to account for the majority of small bowel perforations [2]. In our study, approximately $64 \%$ of traumatic small bowel

Table 3. Treatment results

\begin{tabular}{|lccc|}
\hline & SBI-A (n=91) & SBI-LI (n=58) & $\boldsymbol{p}$-value \\
\hline ICU stay (day) & $6.7 \pm 0.2$ & $11.1 \pm 0.5$ & 0.047 \\
Hospital stay (day) & $26.9 \pm 1.5$ & $33.5 \pm 2.7$ & 0.092 \\
Morbidity & & & \\
$1,2,3$ & $90(98.9)$ & $51(87.9)$ & 0.215 \\
4,5 & $24(26.4)$ & $27(46.6)$ & 0.031 \\
Mortality & $13(14.3)$ & $13(22.4)$ & 0.061 \\
\hline
\end{tabular}

Values are presented as mean \pm standard deviation or number (\%). SBI-A: small bowel injury alone, SBI-Ll: small bowel injury accompanied by liver injury, ICU: intensive care unit. injuries were caused by TAs, followed by falls (26\%), and other causes (10\%).

In the current study, the most frequent injury mechanism was passenger TAs in the SBI-A group, but pedestrian TAs in the SBI-LI group. SBI can be frequently caused by seat-belt injury [10], while LI can be easily caused by direct blunt trauma.

We investigated several prognostic factors, such as AIS scores, the initial hemodynamic status, time between the accident and surgery, operation time, amount of transfusion, and small bowel perforation. Higher AIS scores indicate multiple, severe trauma, which may be associated with more blood transfusions during the operation, a longer ICU stay, and higher morbidity rates $[11,12]$. The current study showed higher AIS, higher amounts of RBCs transfused, and longer ICU stays in the SBI-LI group than in the SBI-A group, which is a reasonable result. Regarding mortality, no significant difference was found. However, a study using a larger number of patients may show a significant difference between groups in this regard.

Initial hypotension is a known significant prognostic factor of blunt trauma $[13,14]$. However, its effect in this study was inconclusive, as no significant between-group difference was noted.

In SBI, surgical delay is known to be a poor prognostic factor [15]. However, it was difficult to elucidate the effect of surgical delay because the results of this study showed no significant difference between the two groups.

Although operation time is considered a prognostic factor for mortality, little research has been published on this matter. In our study, we investigated operation time as a possible factor related to mortality and morbidity. It was nearly identical in both groups, possibly because there were no cases of high-grade LI, and liver-related treatments were therefore almost never required.

There are several surgical techniques for traumatic bowel injury, and the preferred method has changed over the years. In the 1940s, stoma construction was preferred for traumatic bowel injury, but primary repair is now the preferred method [16]. In our study, resection of the injured bowel was the most frequently performed surgical procedure. In cases of bowel perforation or severe damage, primary repair alone may cause complications or require re-operation, which is why bowel resection was 
mainly performed.

In patients with traumatic bowel injury, LI can cause massive bleeding. Some research has investigated the transfusion amount as a prognostic factor in cases of trauma. Mostafa et al. [17] suggested that the Injury Severity Score, Glasgow Coma Scale, and packed-cell transfusion volume were independent predictors of mortality. We found that the SBI-LI group had significantly more RBCs transfused than the SBI-A group, which may have been the result of LI bleeding or other injuries associated with a higher AIS score. This may have affected the difference in morbidity between the two groups, but not the mortality.

The affected organ, such as the small bowel or colon, is related to the degree of peritoneal contamination and the specific risk of surgical intervention [2]. Furthermore, intraperitoneal contamination following bowel perforation can be an important prognostic factor. However, as this study was a retrospective study, we only investigated the presence of small bowel perforation and not the range and grade of contamination.

There are three limitations of this study. First, this study was limited by its retrospective design. In particular, we could not determine the effect of small bowel perforation because it was not possible to analyze the grade of contamination caused by bowel perforation. Second, it was difficult to investigate the true effect of LI because there were not many high-grade LI cases. If more cases of highgrade LI had been included, the results of this study may have been different. Third, this study was performed using data collected from a general hospital and not trauma centers; if data from trauma centers had been analyzed, the patient characteristics, treatments, and study results may have differed.

\section{CONCLUSION}

The SBI-LI group required more RBC transfusions and had longer ICU stays than the SBI-A group. The SBI-LI group had a higher mortality rate than the SBI-A group, although this difference was not statistically significant; thus, larger-scale follow-up research is needed.

\section{REFERENCES}

1. Davis JJ, Cohn I Jr, Nance FC. Diagnosis and management of blunt abdominal trauma. Ann Surg 1976;183:672-8.

2. Fraga GP, Silva FH, Almeida NA, Curi JC, Mantovani M. Blunt abdominal trauma with small bowel injury: are isolated lesions riskier than associated lesions? Acta Cir Bras 2008;23:192-7.

3. Watts DD, Fakhry SM; EAST Multi-Institutional Hollow Viscus Injury Research Group. Incidence of hollow viscus injury in blunt trauma: an analysis from 275,557 trauma admissions from the East multi-institutional trial. J Trauma 2003;54:289-94.

4. Faria GR, Almeida AB, Moreira H, Barbosa E, Correia-da-Silva P, Costa-Maia J. Prognostic factors for traumatic bowel injuries: killing time. World J Surg 2012;36:807-12.

5. Slotta JE, Justinger C, Kollmar O, Kollmar C, Schäfer T, Schilling MK. Liver injury following blunt abdominal trauma: a new mechanism-driven classification. Surg Today 2014;44:241-6.

6. Norrman G, Tingstedt B, Ekelund M, Andersson R. Non-operative management of blunt liver trauma: feasible and safe also in centres with a low trauma incidence. HPB (Oxford) 2009;11:50-6.

7. Kim JW, Kwak SS, Park MK, Koo YP. Clinical aspects and prognostic factors of small bowel perforation after blunt abdominal trauma. J Korean Soc Traumatol 2011;24:82-8.

8. Hoyt DB, Coimbra R, Acosta J. Management of acute trauma. In: Townsend CM, Beauchamp RD, Evers BM, Mattox KL, eds. Sabiston textbook of surgery. 18th ed. Philadelphia:W.B.Saunders;2004:507-8.

9. Vance BM. Traumatic lesions of the intestine caused by nonpenetrating blunt force. Arch Surg 1923;7:197-212.

10. Chandler CF, Lane JS, Waxman KS. Seatbelt sign following blunt trauma is associated with increased incidence of abdominal injury. Am Surg 1997;63:885-8.

11. Croce MA, Fabian TC, Stewart RM, Pritchard FE, Minard G, Kudsk KA. Correlation of abdominal trauma index and injury severity score with abdominal septic complications in penetrating and blunt trauma. J Trauma 1992;32:380-7; discussion 387-8.

12. Bull JP. The Injury Severity Score of road traffic casualties in relation to mortality, time of death, hospital treatment time and disability. Accid Anal Prev 1975;7:249-55.

13. Kafie F, Tominaga GT, Yoong B, Waxman K. Factors related to outcome in blunt intestinal injuries requiring operation. Am Surg 1997;63:889-92.

14. Edubio MN. ASA physical status score as a predictive tool of mortality in emergency postoperative abdominal injuries in the 
Yu Seong Noh, et al. Small Bowel Injury and Liver Injury

ICU. BJMMR 2017;21:1-7.

15. Fakhry SM, Watts DD, Luchette FA; EAST Multi-Institutional Hollow Viscus Injury Research Group. Current diagnostic approaches lack sensitivity in the diagnosis of perforated blunt small bowel injury: analysis from 275,557 trauma admissions from the EAST multi-institutional HVI trial. J Trauma
2003;54:295-306.

16. Maxwell RA, Fabian TC. Current management of colon trauma. World J Surg 2003;27:632-9.

17. Mostafa G, Gunter OL, Norton HJ, McElhiney BM, Bailey DF, Jacobs DG. Age, blood transfusion, and survival after trauma. Am Surg 2004;70:357-63. 